\title{
Three-Dimensional Virtual Reality Simulation to Safe Planning Neurosurgical Procedure in Brain Aneurysms, Latin American Single-Center Experience: Advantages and Limitations
}

\author{
Antonio M. Zárate-Méndez $\quad$ José M. Ramos-Delgado, $\quad$ Juan C. Lujan-Guerra ${ }^{1, \odot}$ \\ Carlos D. Rio-Olivares ${ }^{1} \quad$ Luis E. Moreira-Ponce ${ }^{1} \quad$ José L. Aceves-Chimal ${ }^{1}$
}

${ }^{1}$ Department of Neurosurgery, Centro Médico Nacional "20 de

Address for correspondence Jose Miguel Ramos-Delgado, MD, Av. Noviembre" Instituto de Seguridad y Servicios Sociales de los Felix Cuevas 540 Col. Del Valle, Delegacion Benito Juarez, Mexico Trabajadores del Estado (ISSSTE), Mexico City, Mexico

city, CP 03229 (e-mail: jmiguelrd1@gmail.com).

Indian J Neurosurg 2021;1:69-73.

\begin{abstract}
Background The neurosurgical approach to clipping cerebral aneurysms has been a complex challenge for all neurosurgeon experts in cerebrovascular surgery. The three-dimensional computed tomography angiography (3D-CTA) allows identifying bone and vascular structures close to an aneurysm to simulate in virtual 3D images, the appropriate and safest approach to cerebral aneurysm clipping.

Objectives This study aims to share our experience using 3D simulation as a support to the safe planning for cerebrovascular disease surgery.

Materials and Methods We reviewed the surgical outcomes from a cerebrovascular neurosurgeon using the 3D-CTA images in 360-degree reconstruction in the planning of the preoperative surgical procedure for the treatment of brain aneurysm. In all patients, the virtual surgical approach was replicated in real-time surgery.

Results We analyzed 34 patients around $51 \pm 8$ years of age. Of these, 76.5\% $(n=26)$ and $23.5 \%(n=8)$ were males and females, respectively. Saccular aneurysms were the most frequent ( $85 \%)$, the Arteries affected by aneurysms were middle cerebral artery

Keywords

- cerebral aneurysm

- virtual preoperative surgical approach

- 3D-CTA

- approach simulation

- safe planning surgery $(n=6)$, basilar tip $(n=6)$, vertebral artery in V3 and V4 $(n=6)$, and posterior cerebral artery $(n=5)$. The virtual surgical pterional approach was the most frequently used (50\%), followed by fronto-orbito-zigomático (29\%) and far lateral (15\%) approaches. There were no intraoperative complications in any patient.

Conclusion Preoperative 3D virtual reality simulation is a great support tool to perform a safe surgical procedure in real-time for the treatment of simple and complex brain aneurysms.
\end{abstract}

\section{Introduction}

The neurosurgical approach to clipping cerebral aneurysms has been a difficult challenge for the expert cerebrovascular

DOI https://doi.org/ $10.1055 / \mathrm{s}-0041-1725233$ ISSN 2277-954X. neurosurgeon, forcing the neurosurgeon to combine their surgical skills with their mental map of the brain-vascular anatomy to plan the surgical approach and also have resolved any transsurgical incidental complications. They must face the (c)2021. Neurological Surgeons' Society of India.

This is an open access article published by Thieme under the terms of the Creative Commons Attribution-NonDerivative-NonCommercial-License, permitting copying and reproduction so long as the original work is given appropriate credit. Contents may not be used for commercial purposes, or adapted, remixed, transformed or built upon. (https://creativecommons.org/licenses/by-nc-nd/4.0/). Thieme Medical and Scientific Publishers Pvt. Ltd. A-12, 2nd Floor, Sector 2, Noida-201301 UP, India 
challenge of learning, planning, and performing increasingly complex surgical procedures with little margin for error. ${ }^{1,2}$

The importance of precisely understanding the threedimensionality of aneurysms before clipping has been emphasized in several publications since $1995 .{ }^{3}$ There are many ways to acquire surgical skills through neurosurgical training, animal laboratories, and carcass which have been a standard for learning but they have many practical limitations, and the use of physical models may be equally restricted; therefore, the need to incorporate new techniques to optimize surgical results has grown. ${ }^{2}$ Continuous upgrading of computing and virtual reality environments now offer additional flexibility of training possibilities for users, especially in the training of neurosurgeons in the cerebrovascular specialty. We consider that the variations in surgical effectiveness observed in the past were possibly the result of an inappropriate surgical approach since the different study techniques did not allow virtual reality planning, making the surgical result depend on the experience of the neurosurgeon. ${ }^{4-13}$

With three-dimensional computed tomography angiography (3D-CTA), it has been possible to observe structural details of the cerebral aneurysm, improving the diagnostic sensibility to identify hidden structures and aneurysms smaller than $3 \mathrm{~mm}$, and to perform a preoperative virtual reality planning surgical approach. ${ }^{6-14}$ In our experience, using the 3D-CTA study as a tool for preoperative virtual reality planning with 360-degree image reconstruction, we were able to visualize bone structures and vascular network adjacent to a cerebral aneurysm, also to perform the neurosurgical procedure in real-time, distributing this technique into the highly specialized cerebrovascular program for the training of neurosurgeons. In this document, we describe the results obtained whit this preoperative technique.

\section{Materials and Methods}

We reviewed the surgical outcomes from January 2017 to August 2019 by a neurosurgeon specialized in cerebrovascular surgery, who used only 3D-CTA images with 360-degree reconstruction to preoperative virtual reality planning surgical approach to clipping cerebral aneurysm to apply it into the surgical procedure in real-time. The 3D-CTA study was performed on a 64-slice Siemens scanner, with a record of 300 cuts of $1 \mathrm{~mm}$. All the images and reconstruction of aneurysm were analyzed by a neuroradiologist and by the neurosurgeon in the Picture Archiving and Communication System (PACS) equipment installed in the Apple Mac system using the KDS Kanteron Systems version 3.1b2 in multiple projections simulating the surgical procedure.

In all patients, bone and vascular structures adjacent to the cerebral aneurysm were identified with a potential risk of promoting intraoperative complications. These structures were virtually removed to reach the appropriate site to perform the aneurysm clipping.

The surgical procedure in real-time was performed based on the virtual approach without the need for any modifications during the surgery, achieving a successful aneurysm clipping in all patients (-Fig. 1). Finally, epidemiological

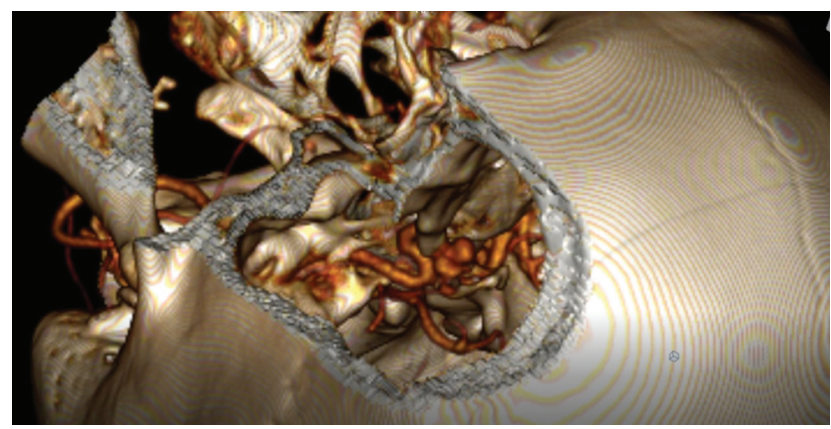

Fig. 1 Virtual pterional approach in bilobulated aneurysm of the posterior communicating segment.

variables, location, and dimensions of the aneurysms, surgical outcome, complications related to contrast, and surgical procedure were recorded from the clinical history.

\section{Statistical Analysis}

The descriptive analysis was performed with percentages and absolute numbers using SPSS statistical package v25.0 for Windows operative software.

\section{Results}

We analyzed 34 patients around $51 \pm 8$ years of age. Of these, 76.5\% $(n=26)$ and $23.5 \%(n=8)$ were males and females, respectively. The initial symptomatology was mainly characterized by headache, motor dysfunction, or disturbance of some cranial nerve. Also, $47 \%$ of patients were in grade I of Fisher's stratification and $60 \%$ in grade I of Hunt-Hess scale (-Table 1). Saccular aneurysms were the most frequent (85\%) with dimensions in the neck, equator, and dome in the rank of 2.6 to 20,16 to 28 , and 1 to $25 \mathrm{~mm}$, respectively. The anatomical location showed a similar proportional distribution in the middle cerebral artery $(n=6)$, basilar tip $(n=6)$, and vertebral artery in V3 and V4 $(n=6)$ followed by posterior cerebral artery ( $n=5$; - Table 2$)$.

The preoperative virtual and surgical approaches were the pterional (50\%), fronto-orbito-zigomático (29\%), and lateral (15\%), examples are shown in - Figs. 1 and 2, and there was no intraoperative complication. Postoperative complications impossible to prevent with preoperative virtual approach but, related with neurological surgery, were observed in eight patients: neurogenic shock $(n=1)$, spinal cerebral fistula $(n=3)$, hygroma $(n=1)$, hydrocephalus $(n=1)$, and cerebral infarction due to vasospasm $(n=1)$. No patients had any complications related to the contrast medium used in the 3D-CTA study (- Table 2 ).

\section{Discussion}

Neurosurgeons, in particular, are faced with the challenge of learning, planning, and performing complex surgical procedures and, with continuous improvements, virtual reality now offers the potential for flexible training and preoperative experiences. ${ }^{15}$ The preoperative planning of surgical approaches to clipping cerebral aneurysms is an 
indispensable mental process that neurosurgeons must perform to achieve a safer surgical act, prevent inadvertent or incidental injuries during the intervention, and, of course, to obtain better results. Before 3D-CTA, the cerebrovascular neurosurgeon had to plan the surgical procedure only with mental images obtained from his experience; however, intraoperative improvisation to avoid lesions to the bone and vascular structures near to cerebral aneurysm frequently have to be done. Since 1995, 3D-CTA had been used in surgical planning with its technological limitations in that time, ${ }^{3}$ in fact, with 3D-CTA reconstructed in 360 degrees, it is possible to virtually eliminate the cerebral content, bone structures, and vessels adjacent to the cerebral aneurysm, allowing to

Table 1 Clinical symptomatology and neurological assessment

\begin{tabular}{|c|c|c|}
\hline & $n$ & $\%$ \\
\hline Headache/migraine & 13 & 38 \\
\hline Motor dysfunction & 8 & 23 \\
\hline Cranial nerve disorder & 8 & 23 \\
\hline Cerebral stroke & 6 & 17 \\
\hline \multicolumn{3}{|l|}{ Hunt-Hess scale } \\
\hline I & 16 & 47 \\
\hline II & 4 & 12 \\
\hline III & 4 & 12 \\
\hline IV & 10 & 29 \\
\hline \multicolumn{3}{|l|}{ Fisher's scale } \\
\hline I & 19 & 55 \\
\hline II & 5 & 15 \\
\hline III & 2 & 6 \\
\hline IV & 5 & 15 \\
\hline V & 3 & 9 \\
\hline
\end{tabular}

observe in different projections, exclusively the cerebral aneurysm, making it virtually easier for the neurosurgeon to select a better surgical approach with the lowest risk of intraoperative complications and the specific clip according to each type of cerebral aneurysm.

Table 2 Anatomical Location, cerebral aneurysms types, surgical approach and early postoperative complication

\begin{tabular}{|c|c|c|}
\hline & $n$ & $\%$ \\
\hline \multicolumn{3}{|l|}{ Aneurysm types } \\
\hline Sacular & 29 & 85 \\
\hline Fusiform & 5 & 15 \\
\hline \multicolumn{3}{|l|}{ Anatomical location } \\
\hline Middle cerebral artery & 6 & 17 \\
\hline Basilar artery & 6 & 17 \\
\hline Cerebral posterior artery & 5 & 14 \\
\hline $\begin{array}{l}\text { Vertebral artery } \\
\text { (V3 and V4 segments) }\end{array}$ & 6 & 17 \\
\hline Others $^{\mathrm{a}}$ & 11 & 32 \\
\hline \multicolumn{3}{|l|}{ Surgical approach } \\
\hline Peterional & 17 & 50 \\
\hline Fronto-orbito-zigomático & 10 & 29 \\
\hline Far lateral approach & 5 & 15 \\
\hline Retrosigmoid & 2 & 3 \\
\hline \multicolumn{3}{|c|}{ Postoperative neurological complications } \\
\hline Neurogenic shock & 3 & 9 \\
\hline Spinal cerebral fistula & 3 & 9 \\
\hline Hygroma & 1 & 3 \\
\hline Hydrocephalus & 1 & 3 \\
\hline
\end{tabular}

aphthalmic artery, basilar trunk, right labyrinth, communicating segment, vertebrobacillary union.
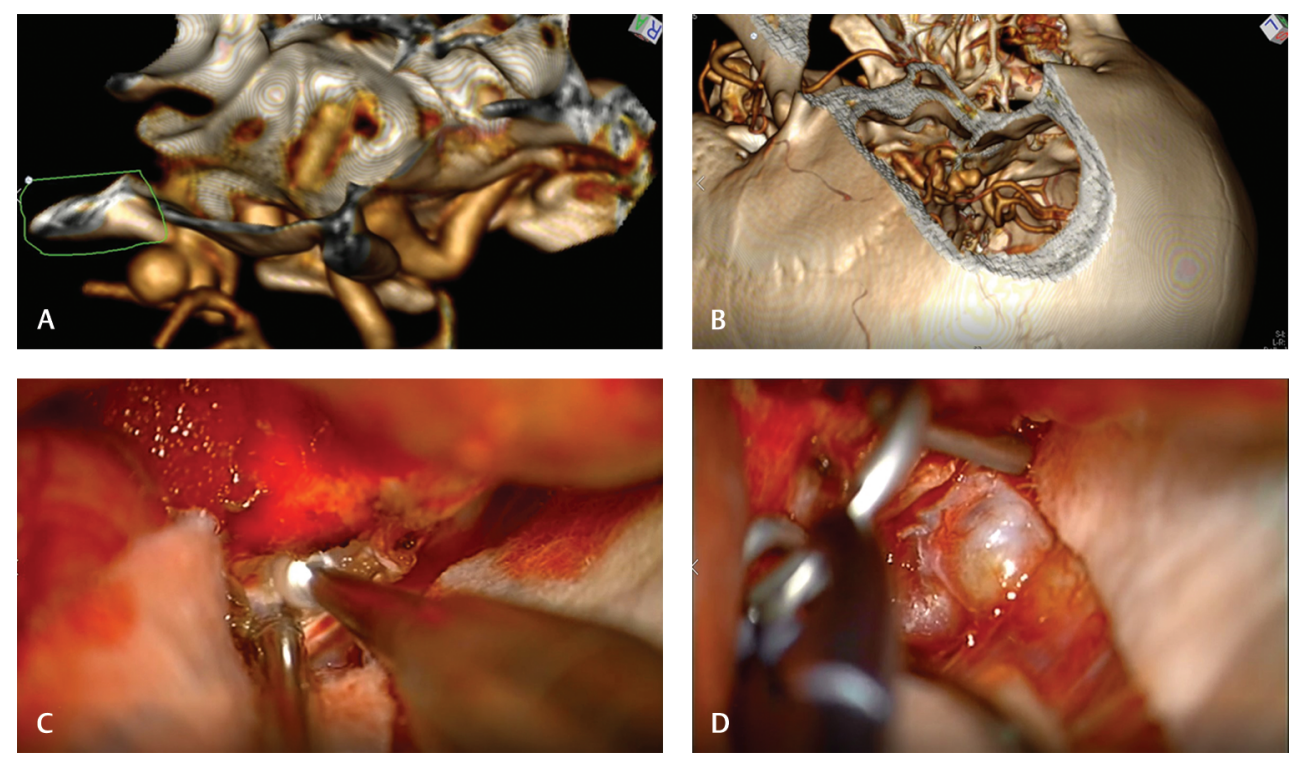

Fig. 2 (A) Virtual clinoidectomy, (B) final virtual view, (C) real clinoidectomy, and (D) aneurysm clipping. 
In the Neurosurgery Department of our institution, we are using this technique before the surgical procedure, applying it in surgery in real-time, reaching in all cases successful aneurysm clipping without incidental intraoperative injuries or neurological complications, despite having performed surgical approaches in arterial segments with difficult access. These findings contrast with those reported in the international medical literature, where the planning of surgical approach is performed only with images obtained from the contrasted two-dimensional (2D) study, observing an incidence of intraoperative complications between 12 and $20 \%$, $8,14,16-24$

We observed that with the 3D-CTA, 360-degree reconstruction offered an additional advantage, which consists in the possibility of eliminating brain mass, bone, and vascular structures that have allowed us to perform a preoperative virtual reality surgical approach and after apply it in real-time surgery, with successful results in cerebral aneurysm clipping, as well as the teaching of this virtual technique in the cerebrovascular training program, for neurosurgeons.

Another challenge is to visualize a surgical approach to the mental construct that can predict what will be encountered in the operating room. For this reason, considerable research effort has gone into developing methods and systems that are capable of fusing multimodal imaging data and presenting the surgically relevant information in interactive 3D displays. ${ }^{25}$

In the field of surgical practice, greater improvement in programs and virtual immersion has been applied, an example of this is the Immersive Touch Aneurysm Clipping Simulator (ITACS) which provide realistic haptic virtual reality clipping of a patient-based cerebral aneurysm. The system provides force feedback simultaneously in real time. ${ }^{26}$ Although we are aware of this kind of technology, there are only a few first world countries which count on this technology. Unfortunately in developing countries, there is no access to this technology.

In summary, the preoperative 3D virtual surgical approach with 360-degree reconstruction to plan the surgical procedure in real time used together with the cognitive skills and experience of cerebrovascular neurosurgeon allows greater efficiency with any incidental transsurgical complication in our institution, also practicing surgeons can potentially review unusual procedures and challenging anatomy in a safe and simulated environment. Even experts can plan and test complex approaches and assess the advantages of various approaches on a virtual representation of a patient's specific anatomy. Meanwhile, as costs decline and the technology matures, virtual reality and augmented reality are sure to play an increasingly important role in cerebrovascular neurosurgery in the years and decades to come.

\section{Conclusion}

Although 3D-CTA is a cheap diagnostic tool that is used in many medical centers, some of them do not use it as a safety planning tool for clipping aneurysms. We try to demonstrate how despite the absence of digital angiography or magnetic resonance, CTA offers many advantages in cerebrovascular disease. The preoperative 3D virtual reality simulation is a good support tool to perform a safe surgical procedure in real-time for the treatment of simple and complex brain aneurysms. With the improvement and evolution of virtual reality, 3D-CTA offers a cheap and low-risk study that can be considered as a gold-standard study in the diagnosis of cerebral aneurysms.

\section{Conflict of interest}

None declared.

\section{Funding}

None.

\section{References}

1 Amacher AL, Drake CG. Aneurysm surgery in the seventh decade. In: Fusek I, Kunc Z, eds. Present Limits of Neurosurgery-Proceedings of the Fourth European Congress of Neurosurgery. June 28-July 2, 1971, in Prague. Prague: Czechoslovak Medical Press; 1972

2 Chan S, Conti F, Salisbury K, Blevins NH. Virtual reality simulation in neurosurgery: technologies and evolution. 2013;72(suppl-1):154-164

3 Harbaugh RE, Schlusselberg DS, Jeffery R, et al. Threedimensional computed tomographic angiography in the preoperative evaluation of cerebrovascular lesions. Neurosurgery 1995;36(2):320-326, discussion 326-327

4 Bull JWB. Contribution of radiology to the study of intracranial aneurysms. BMJ 1962;2(5321): :1701-1708

5 Matsumoto M. Three dimensional computerized tomography-angiography guide surgery of acutely ruptured cerebral aneurysm. J Neurosurg 2001;94(5):718-727

6 Nagai M, Watanabe E. Benefits of clipping surgery based on three-dimensional computed tomography angiography. Neurol Med Chir (Tokyo) 2010;50(8):630-637

7 Xing W, Chen W, Sheng, J, et al. Sixty-four-row multislice computed tomographic angiography in the diagnosis and characterization of intracranial aneurysm: comparison with 3D rotational angiography. World Neurosurg 2011;76(1,2): 105-113

8 Alberico RA, Patel M, Casey S, Jacobs B, Maguire W, Decker R. Evaluation of the circle of Willis with three-dimensional CT angiography in patients with suspected intracranial aneurysms. AJNR Am J Neuroradiol 1995;16(8):1571-1578, discussion $1579-1580$

9 Anderson GB, Steinke DE, Petruk KC. Computer tomography angiography versus digital subtraction angiography for the diagnosis and early treatment of ruptured intracranial aneurysms. Neurosurgery 1999;45(6):1320-1322

10 Chappel ET, Moure FC, Good MC. Comparison of computed tomographic angiography with digital subtraction angiography in the diagnosis of cerebral aneurysms: a meta-analysis. Neurosurgery 2003;52(3):624-631

11 Méndez AMZ, Rosas NPPGuerra JCL, et al. Computed tomography angiography with three-dimensional reconstruction versus rotational angiography as a screening test in patients wilth suspected cerebral aneurysm. Indian J Neurosurgery 2016;5(2):95-100

12 Ligon BL. Biography: history of developments in imaging techniques: Egas Moniz and angiography. Semin Pediatr Infect Dis 2003;14(2):173-181

13 Zhang H, Hou C, Zhou Z, Zhang H, Zhou G, Zhang G. Evaluating of small intracranial aneurysms by 64-detector CT angiography: 
a comparison with 3-dimensional rotation DSA or surgical findings. J Neuroimaging 2014;24(2):137-143

14 Pradilla G, Wicks RT, Hadelsberg U, et al. Accuracy of computed tomography angiography in the diagnosis of intracranial aneurysms. World Neurosurg 2013;80(6):845-852

15 Chan S, Conti F, Salisbury K, Blevins NH. Virtual reality simulation in neurosurgery: technologies and evolution. Neurosurgery 2013;72(suppl 1):154-164

16 Kumar A, Kato Y, Motoharu H, et al. An update on three-dimensional ct angiography in aneurysms: a useful modality for a neurosurgeon. Turk Neurosurg 2013;23(3):304-311

17 Mo DP, Bao SD, Li L, Yi ZQ, Zhang JY, Zhang Y. Virtual reality system for diagnosis and therapeutic planning of cerebral aneurysms. Chin Med J (Engl) 2010;123(16):2206-2210

18 Kumar A, Kato Y, Motoharu H, et al. An update on three-dimensional ct angiography in aneurysms: a useful modality for a neurosurgeon. Turk Neurosurg 2013;23(3):304-311

19 Safavi-Abbasi S, Moron F, Sun H, et al. Techniques and longterm outcomes of cotton-clipping and cotton-augmentation strategies for management of cerebral aneurysms. J Neurosurg 2016;125(3):720-729

20 He H, Chen C, Li W, et al. Contralateral approach based on a preoperative 3-dimensional virtual osteotomy technique for anterior circulation aneurysms. J Stroke Cerebrovasc Dis 2019;28(4):1099-1106
21 Chivukula VK, Levitt MR, Clark A, et al. Reconstructing patient-specific cerebral aneurysm vasculature for in vitro investigations and treatment efficacy assessments. J Clin Neurosci 2019;61:153-159

22 Goertz L, Kabbasch C, Borggrefe J, et al. Preoperative three-dimensional angiography may reduce ischemic complications during clipping of ruptured intracranial aneurysms. World Neurosurg 2018;120:e1163-e1170

23 Cebral JR, Fernando Mut F, GadeP, et al. Combining data from multiple sources to study mechanisms of aneurysm disease: tools and techniques. Int J Numer Methods Biomed Eng 2018;34(11):e3133

24 Matsukawa H, Kamiyama H, Miyazaki T, et al. Surgical treatment of middle cerebral artery aneurysms: aneurysm location and size ratio as risk factors for neurologic worsening and ischemic complications. World Neurosurg 2018;117:e563-e570

25 Liu X, Tao H, Xiao X, et al. Use of the stereoscopic virtual reality display system for the detection and characterization of intracranial aneurysms: a Icomparison with conventional computed tomography workstation and 3D rotational angiography. Clin Neurol Neurosurg 2018;170:93-98

26 Alaraj A, Luciano CJ, Bailey DP, et al. Virtual reality cerebral aneurysm clipping simulation with real-time haptic feedback. Neurosurgery 2015;11 suppl 2(02):52-88 